Article

\title{
Making of Massoia Lactone-Loaded and Food-Grade Nanoemulsions and Their Bioactivities against a Pathogenic Yeast
}

\author{
Li Yuan ${ }^{1}$, Hong-Qian Zhang ${ }^{1}$, Zhe Chi ${ }^{1,2} \mathbb{D}$, Guang-Lei Liu ${ }^{1,2} \mathbb{D}$ and Zhen-Ming Chi ${ }^{1,2, *}$ \\ 1 College of Marine Life Science, Ocean University of China, Yushan Road, No. 5, Qingdao 266003, China; \\ ohnoyuan@163.com (L.Y.); 18801586@163.com (H.-Q.Z.); cz1108@ouc.edu.cn (Z.C.); liugl@ouc.edu.cn (G.-L.L.) \\ 2 Laboratory for Marine Biology and Biotechnology, Qingdao National Laboratory for Marine Science and \\ Technology, Qingdao 266003, China \\ * Correspondence: chi@ouc.edu.cn; Tel.: +86-532-82032266
}

check for updates

Citation: Yuan, L.; Zhang, H.-Q.; Chi, Z.; Liu, G.-L.; Chi, Z.-M. Making of

Massoia Lactone-Loaded and Food-Grade Nanoemulsions and Their Bioactivities against a Pathogenic Yeast. J. Mar. Sci. Eng. 2022, 10, 339. https://doi.org/ $10.3390 /$ jmse10030339

Academic Editors: Francesco

Tiralongo, Gioele Capillo

and Armando Macali

Received: 28 January 2022

Accepted: 20 February 2022

Published: 1 March 2022

Publisher's Note: MDPI stays neutral with regard to jurisdictional claims in published maps and institutional affiliations.

Copyright: (C) 2022 by the authors. Licensee MDPI, Basel, Switzerland. This article is an open access article distributed under the terms and conditions of the Creative Commons Attribution (CC BY) license (https:// creativecommons.org/licenses/by/ $4.0 /)$.

\begin{abstract}
Nanoemulsions (NEs) have been made for improving the delivery and disperse of bioactive compounds. In this study, it was found that the best ingredients for the stable Massoia lactoneloaded and food-grade NEs making were $560.0 \mu \mathrm{L}$ of Tween-80, $240.0 \mu \mathrm{L}$ of Span-80 and $200.0 \mu \mathrm{L}$ of Massoia lactone. Then, $9.0 \mathrm{~mL}$ of distilled water was titrated into the mixture under continuous magnetic stirring (750 rotations $\mathrm{min}^{-1}$ ) with about 2 drops per second for $20 \mathrm{~min}$. Finally, the system was treated by ultrasonication using an ultrasonic generator $(180 \mathrm{~W}$ and $22 \mathrm{KHz}$ ) for $5 \mathrm{~min}$. All the prepared particles with a mean droplet diameter of $43 \mathrm{~nm}$ were spherical, had uniform size distribution and were equally distributed in the Massoia lactone-loaded NEs. The obtained Massoia lactone-loaded nanoemulsions (NEs) were very stable without changes of the mean droplet diameter and polydispersity indexes (PDI) for over two months under different conditions. As with free Massoia lactone, Massoia lactone loaded in the NEs had high anti-fungal activity against Metschnikowia bicuspidate LIAO, a pathogenic yeast causing milky disease in the Chinese mitten crab by damaging its cell membrane and causing cellular necrosis. Massoia lactone loaded in the NEs also had the DPPH radical scavenging activity and the hydroxyl radical scavenging activity.
\end{abstract}

Keywords: liamocin; marine derived Aureobasidium; massoia lactone loaded NEs; anti-fungal activity; anti-oxidant activity

\section{Introduction}

Massoia lactone used in this study is an $\alpha, \beta$-unsaturated $\delta$-lactone moiety substituted at the C6 position by an alkyl chain of 5 carbons. Such a Massoia lactone is designated as C-10 Massoia lactone. It can be released from liamocins synthesized and secreted by the marine-derived Aureobasidium spp. and it has been clear how to biosynthesize liamocins at the molecular level [1]. Because such Massoia lactone has many bioactivities and can be used as a flavoring and fragrant agent, it can be widely applied to food and flavor industries [1], indicating that it is safe to humans. In recent years, it has been found that a large amount of Massoia lactone can be obtained by hydrolysis of liamocins which are synthesized by different strains of marine-derived Aureobasidium spp. and are composed of a single mannitol or arabitol or xylitol headgroup linked to either three or four or even six 3,5-dihydroxydecanoic ester tail-groups [2,3]. Under alkaline conditions, each molecule of liamocins can be hydrolyzed into mannitol or arabitol and three or four or even six 3,5dihydroxydecanoic acid. In turn, 3,5-dihydroxydecanoic acid can be transformed into C-10 Massoia lactone under the acidic condition [4]. It has been reported that $43.04 \pm 1.2 \mathrm{~g} \mathrm{~L}^{-1}$ of extracellular liamocins can be produced and secreted by a marine-derived yeast-like fungus Aureobasidium melanogenum M39 expressing homologous PYC1 gene encoding pyruvate carboxylase and alkaline hydrolysis of the extracellular liamocins can release $39.6 \mathrm{~g} \mathrm{~L}^{-1}$ of 
5-hydroxy-2-decenoic acid lactone into medium [5]. Much research work has demonstrated that C-10 Massoia lactone has antifungal, antiviral, anticancer, and anti-inflammatory activities, suggesting that it could be applied to many sectors of agricultural, industrial, pharmaceutical and food industries [1,6-9]. For example, the treatment of the pathogenic yeast strain LIAO causing the milky disease in the Chinese mitten, an important food in China and other countries with Massoia lactone can damage its cell membrane, even break its whole cells and cause its cellular necrosis [4]. In fact, Massoia lactone displays strong antifungal properties against a broad range of fungal spores, animal and human pathogens, and even harmful insects [1]. Because it is a safe and biodegradable organic natural product with high yield, the high anti-fungal activity and the multiplicity of mode of its action, Massoia lactone can be a promising candidate for development as an effective, green and friendly bio-fungicide in food industries, avoiding the use of chemical fungicide in agricultural and food industries and reducing environmental pollution [1]. However, the insolubility and weak volatility of Massoia lactone seriously limit its applications. For example, it is difficult to use it directly as the bio-fungicide in food, medical and agricultural and aquaculture industries. As a result, various delivery systems using nanotechnology for improving the stability, solubility, high activity and bioavailability of Massoia lactone are required. Nanoemulsions (NEs) can be easily used for improving the delivery and disperse of bioactive compounds such as Massoia lactone [10]. They have a droplet size ranging from 10 to $500 \mathrm{~nm}$, are kinetically stable, and transparent or slightly turbid systems that usually consist of surfactant, oil, and water [11]. Therefore, the NEs are frequently utilized as carriers of low-water-soluble substances as well as volatile oils. It has been reported that NEs (oil-in-water) are one of such delivery and dispersal systems because of their high stability, solubility in water, high bioavailability, high bioactivity, easily controlled release of bioactive compounds and ease of processing in practice [12]. In this study, water phase, oil (Massoia lactone) phase and a mixture of surfactants were optimized for Massoia lactone-loaded NEs formulation, the prepared NEs were characterized and bioactivities of the prepared NEs were examined.

\section{Materials and Methods}

\subsection{The Fungal Strains and Materials}

The fungal strains used in this study were Metschnikowia bicuspidate LIAO, a pathogenic yeast causing milky disease in the Chinese mitten crab [4] and the marine-derived yeastlike fungal strain A. melanogenum M39 overexpressing the pyruvate carboxylase-encoding PYC1 gene which was used to produce a high level of liamocins [5]. All the yeast strains were cultivated on a YPD medium [4] at $28{ }^{\circ} \mathrm{C}$ for two days. A liamocin production medium consisted of $140.0 \mathrm{~g} \mathrm{~L}^{-1}$ glucose, $0.6 \mathrm{~g} \mathrm{~L}^{-1} \mathrm{NH}_{4} \mathrm{NO}_{3}, 0.1 \mathrm{~g} \mathrm{~L}^{-1} \mathrm{KH}_{2} \mathrm{PO}_{4}, 0.5 \mathrm{~g} \mathrm{~L}^{-1}$ $\mathrm{KCl}$, and $0.2 \mathrm{~g} \mathrm{~L}^{-1} \mathrm{MgSO}_{4} \cdot 7 \mathrm{H}_{2} \mathrm{O}$ and $0.2 \mathrm{~g} \mathrm{~L}^{-1}$ corn steep liquor [5]. Massoia lactone used in this study was obtained and purified from the produced liamocins as described by Zhang et al. [4]. Polyoxyethylene castor oil, Tween-20, Tween-80 and Span-80 which were analytical reagents were purchased from Sinopharm Chemical Reagents Co., Ltd., Shanghai, China.

\subsection{Preparation of Massoia Lactone-Loaded and Food-Grade Nanoemulsions (NEs)}

For every emulsion, first, $560.0 \mu \mathrm{L}$ of Tween-80, $240.0 \mu \mathrm{L}$ of Span-80 and $200.0 \mu \mathrm{L}$ of Massoia lactone were mixed together and the mixture was magnetically stirred for $20 \mathrm{~min}$ (750 rotations $\left.\min ^{-1}\right)$. Then, distilled water $(9.0 \mathrm{~mL}$ ) was added to the organic phase with a speed of about 2 drops of distilled water per second under continuous magnetic stirring (750 rotations $\left.\min ^{-1}\right)$. Afterwards, the system was treated by ultrasonication using an ultrasonic generator $(180 \mathrm{Q}$ and $22 \mathrm{KHz})$ for $5 \mathrm{~min}$. Finally, the stable Massoia lactone-loaded NEs were formed.

In addition, any single surfactant $(800.0 \mu \mathrm{L}$ of polyoxyethylene castor oil or Tween-20, Tween-80 or Span-80) we also tried to form NEs with $200.0 \mu \mathrm{L}$ of Massoia lactone. However, only the mixture $(800 \mu \mathrm{L})$ of Tween-80 and Span-80 could form the stable NEs with $200 \mu \mathrm{L}$ 
of Massoia lactone. Therefore, effects of different volume ratios (9:1, 8:2, 7:3, 6:4, 5:5, 4:6, 3:7, 2:8 and 1:9) of Tween-80 to Span-80 on the stable NE formation and effects of different volume ratios (1:9, 2:8, 3:7, 4:6, 5:5, 6:4, 7:3, 7.5:2.5, 8:2, 8.5:1.5 and 9:1) of the mixture (7:3) of the surfactants to Massoia lactone on the stable NE formation were examined. At the same time, effects of ultrasonic treatment time $(3,5$ and $7 \mathrm{~min})$ on the stable NE formation were also tested. The formed stable NEs were photographed and the Tyndall phenomenon in the NEs was checked using a laser pointer.

\subsection{Transmission Electron Microscopy}

One droplet of the stable Massoia lactone-loaded NEs was added to a copper grid and the copper grid was dried on a filter paper. After drying, one droplet of phosphotungstic acid $\left(20.0 \mathrm{~g} \mathrm{~L}^{-1}\right)$ was added to the copper grid to stain the NE particles for 1-2 min. The stained NE particles were observed and photographed using a Transmission Electron Microscope (TEM) (JEM-1200EX, JEOL, Tokyo, Japan). The images, morphology and size of the particles were recorded and calculated under the TEM.

\subsection{Measurements of Mean Droplet Diameters and Polydispersity Indexes}

The mean droplet diameters and polydispersity indexes (PDI) of the stable Massoia lactone-loaded NEs were measured using a Zetasizer Nano ZS (Malvern Instruments Ltd., Worcestershire, UK) at room temperature $\left(26^{\circ} \mathrm{C}\right)$ as described in the Instruction Sheet.

\subsection{Physical and Chemical Stability of the Massoia Lactone-Loaded NEs}

The Massoia lactone-loaded NEs made above were utilized for the measurement of physical and chemical stability. The physical and chemical stability of the NEs was evaluated based on the determinations as described below.

The Massoia lactone-loaded NEs were stored at room temperature for 7, 15, 30 and 45 days, respectively, and centrifuged at $4000 \times g$ for $15 \mathrm{~min}$. It must be observed whether or not pellets in the centrifuge tube were formed after each centrifugation. If there were pellets, this meant the system was not stable. The Massoia lactone-loaded NEs were also diluted for 2, 5, 8, 10 and 12 folds. The dilutes were further stored for one week. Then, the droplet diameters and PDI in the NEs were monitored as described above. After the NEs were stored at $4{ }^{\circ} \mathrm{C}, 25^{\circ} \mathrm{C}, 30^{\circ} \mathrm{C}, 37^{\circ} \mathrm{C}$ and $50^{\circ} \mathrm{C}$ for 0 days, 15 days and 30 days, the droplet diameters and PDI in the NEs were also checked and measured as described above.

\subsection{Anti-Fungal Activity of the Massoia Lactone-Loaded NEs}

M. bicuspidate LIAO, the pathogenic yeast [4] was aerobically cultivated in the YPD liquid medium at $28{ }^{\circ} \mathrm{C}$ and $180 \mathrm{rpm}$ for $10-14 \mathrm{~h}$. After suitable dilution of the culture with sterile distilled water, the dilutes were spread on the YPD plates. $5.0 \mu \mathrm{L}$ of the Massoia lactone-loaded NEs containing $20.0 \mathrm{mg} \mathrm{mL}^{-1}$ of Massoia lactone was added to the sterile filter disc and the disc was placed on the YPD plates which had been inoculated with M. bicuspidate LIAO. At the same time, $5.0 \mu \mathrm{L}$ of $20.0 \mathrm{mg} \mathrm{mL}^{-1}$ of free Massoia lactone (positive control), Tween-80 (negative control) and Span-80 (negative control) was also added to the sterile filter discs and the discs were placed on the same YPD plates which had been inoculated with M. bicuspidate LIAO. All the plates were incubated at $28^{\circ} \mathrm{C}$ for 2-3 days. The clear zones on the plates were observed and photographed and the size of the clear zones was measured. At the same time, the Massoia lactone-loaded NEs and free Massoia lactone stored at $4{ }^{\circ} \mathrm{C}, 25^{\circ} \mathrm{C}$ and $30^{\circ} \mathrm{C}$ for 30 and 60 days were also used for the determination of their anti-fungal activity.

\subsection{The Treated and Untreated Cells Were Stained with PI and the Annexin V-FITC}

One hundred microliters of the treated cells of the pathogenic yeast strain LIAO $\left(0.2 \times 10^{6}\right.$ cells $\left.\mathrm{mL}^{-1}\right)$ using the free Massoia lactone $\left(20.0 \mathrm{mg} \mathrm{mL}^{-1}\right)$ and the Massoia lactone-loaded NEs (containing $20.0 \mathrm{mg} / \mathrm{mL}$ of Massoia lactone) for $6 \mathrm{~h}$ were mixed with $0.05 \mathrm{mg} \mathrm{mL}^{-1}$ of propidium iodide (PI) and the mixtures were incubated at $37^{\circ} \mathrm{C}$ and 
dark for $10 \mathrm{~min}$. The treated cells were observed and photographed under the Olympus U-LH100HG fluorescent microscope. The untreated pathogenic yeast strain LIAO cells $\left(0.2 \times 10^{6}\right.$ cells $\left.\mathrm{mL}^{-1}\right)$ were used as the negative controls.

It has been well confirmed that the Annexin V-FITC apoptosis detection kit can be successfully utilized to test yeast and fungal cellular apoptosis or cellular necrosis [13]. The same yeast cells treated and untreated as described above were centrifuged at $10,000 \times g$ for $6 \mathrm{~min}$ and the pellets obtained were washed with a sterile phosphate buffer ( $\mathrm{pH}$ 7.0) 3 times by centrifugation at $10,000 \times g$ for $8 \mathrm{~min}$. The washed yeast cell pellets were suspended in $600.0 \mu \mathrm{L}$ of the Annexin V-FITC binding solution of the kit and the cell suspension was mixed well with $5.0 \mu \mathrm{L}$ of the Annexin V-FITC of the kit. Then, $10.0 \mu \mathrm{L}$ of PI solution was mixed well with the cell suspension. The new cell suspension was kept in the dark for $15 \mathrm{~min}$. After that, the new cell suspension was immediately incubated in the ice in the dark. The cell suspension was observed and photographed using the Olympus U-LH100HG fluorescent microscope and the images were recorded within one hour.

\subsection{Determination of Antioxidant Activity}

The aliquot $(2.0 \mathrm{~mL})$ of 2,2-diphenyl-1-picrylhydrazyl radical (DPPH) solution $(0.08 \mathrm{mM}$, dissolved in ethanol) was mixed in a $5.0-\mathrm{mL}$ test tube with $2.0 \mathrm{~mL}$ of Massoia lactone $\left(20 \mathrm{mg} / \mathrm{mL}\right.$, dissolved in ethanol) or $2.0 \mathrm{~mL}$ of the Massoia lactone-loaded NEs $\left(20.0 \mathrm{mg} \mathrm{mL}^{-1}\right.$ of Massoia lactone) dissolved in the same solvent as DPPH. The mixtures were vigorously shaken for $30 \mathrm{~min}$ in the dark and $\mathrm{OD}_{517 \mathrm{~nm}}$ values (A1) of the mixtures were recorded immediately. Two milliliters of ethanol were mixed in the 5.0-mL test tube with $2.0 \mathrm{~mL}$ of Massoia lactone (20.0 mg mL$~^{-1}$ of Massoia lactone) or $2.0 \mathrm{~mL}$ of the Massoia lactone-loaded NEs containing the same concentration of Massoia lactone, respectively. The mixtures were vigorously shaken for $30 \mathrm{~min}$ in the dark and the $\mathrm{OD}_{517 \mathrm{~nm}}$ value (A2) of the mixtures was assayed, immediately. Two milliliters of the mixture (water, Tween- 80 and span 80 ) or $2.0 \mathrm{~mL}$ of ethanol and $2.0 \mathrm{~mL}$ of the DPPH solution $(0.08 \mathrm{mM}$, dissolved in ethanol) were added to the $5.0 \mathrm{~mL}$-test tube. The mixtures were vigorously shaken for $30 \mathrm{~min}$ in the dark and $\mathrm{OD}_{517 \mathrm{~nm}}$ values (A3) of the mixtures were monitored, immediately. The DPPH radical scavenging activity of the sample was calculated based on the following Equation (1) [14]:

$$
\text { DPPH scavenging rate }(\%)=1-(\mathrm{A} 1-\mathrm{A} 2) / \mathrm{A} 3 \times 100 \%
$$

\subsection{Assay of Scavenging Rate of Hydroxyl Radical}

Two milliliters of the Massoia lactone-loaded NEs, or $2.0 \mathrm{~mL}$ of free Massoia lactone (20 mg mL $\mathrm{m}^{-1}$ of Massoia lactone dissolved in ethanol), $700.0 \mu \mathrm{L}$ of $3.0 \% \mathrm{H}_{2} \mathrm{O}_{2}$ solution, $1.0 \mathrm{~mL}$ of $\mathrm{FeSO}_{4}$ solution $(1.5 \mathrm{mM})$ and $300.0 \mu \mathrm{L}$ of salicylic acid solution $(20.0 \mathrm{mM}$, dissolved in ethanol) were mixed thoroughly and the mixture was incubated at $37^{\circ} \mathrm{C}$ for $30 \mathrm{~min}$. The $\mathrm{OD}_{517 \mathrm{~nm}}$ value of the mixture was determined at $517 \mathrm{~nm}$ using a spectrophotometer, immediately and was recorded as A1. Two milliliters of the Massoia lactone-loaded NEs or $2.0 \mathrm{~mL}$ of the free Massoia lactone $(20 \mathrm{mg} / \mathrm{mL}$ of Massoia lactone dissolved in ethanol), $700.0 \mu \mathrm{L}$ of $3.0 \% \mathrm{H}_{2} \mathrm{O}_{2}$ solution, $1.0 \mathrm{~mL}$ of $\mathrm{FeSO}_{4}$ solution $(1.5 \mathrm{mM})$ and $300.0 \mu \mathrm{L}$ of ethanol were mixed thoroughly and the mixture was incubated at $37^{\circ} \mathrm{C}$ for $30 \mathrm{~min}$. The $\mathrm{OD}_{517 \mathrm{~nm}}$ value of the mixture was determined at $517 \mathrm{~nm}$, immediately and was recorded as A2. Two milliliters of the mixture (water, Tween- 80 and Span-80), $700.0 \mu \mathrm{L}$ of $3.0 \%$ $\mathrm{H}_{2} \mathrm{O}_{2}$ solution, $1.0 \mathrm{~mL}$ of $\mathrm{FeSO}_{4}$ solution $(1.5 \mathrm{mM})$ and $300.0 \mu \mathrm{L}$ of salicylic acid solution ( $20.0 \mathrm{mM}$, dissolved in ethanol) were mixed thoroughly and the mixture was incubated at $37^{\circ} \mathrm{C}$ for $30 \mathrm{~min}$. The $\mathrm{OD}_{517 \mathrm{~nm}}$ value of the mixture was determined at $517 \mathrm{~nm}$, immediately and was recorded as A3. The scavenging rate of hydroxyl radical was calculated according to the following Equation (2) [14]: 


\section{Results and Discussion}

\subsection{Preparation of the Massoia Lactone-Loaded and Food-Grade NEs}

The NEs mentioned above have many merits over conventional emulsions such as high stability, obvious increment in the interfacial area, rapid absorption by internalization into the enterocyte, high ability to enhance bioactive compound solubility and bioavailability. The NEs also have high solubilization capacity for both hydrophilic and hydrophobic active compounds due to the presence of the emulsifier-based interface between oil and water. Based on the components, there are three types of the NEs: oil in water $(\mathrm{O} / \mathrm{W})$; water in oil (W/O) and bi-continuous/multiple emulsion [15]. In this study, the O/W type NEs where oil phases (Massoia lactone) were dispersed in water phases (distilled water) were made. It has been reported that nonionic surfactants such as Tween and Span with a hydrophile lipophilic balance (HLB) value greater than 8 could be successfully used to make the $\mathrm{O} / \mathrm{W}$ type NEs [15]. So, based on these, different surfactants such as Tween- 80 or Tween-20 or polyoxyethylene castor oil or Span- 80 were tried to be used to make the O/W type NEs in this study. However, it was found that any single surfactant such as Tween-80 or Tween-20 or polyoxyethylene castor oil or Span-80 could not form stable and suitable NEs with Massoia lactone based on the transparency, particle size, the Tyndall phenomenon, phase separation and fluidity of the prepared NEs. For example, the NEs containing Tween-80 (HLB value was 15) or Tween-20 (HLB value was 16.7) with Massoia lactone had a particle size more than $100 \mathrm{~nm}$. The mixture containing polyoxyethylene castor (HLB value was 14-16) and Massoia lactone formed a gel. The mixture having Span-80 (HLB value was 4.3) and Massoia lactone could not form emulsion and Span- 80 was easily separated from Massoia lactone within $5 \mathrm{~min}$ (data not shown). In contrast, it has been reported that plant essential oils Eugenol (EUG), Carvacrol (CAR) and Cinnamaldehyde (CA) and Tween 80 can form NEs in the aqueous phase [16]. So, in this study, the various surfactant combinations (polyoxyethylene castor + Span-80, Tween-80 + Span-80, Tween-20 + Span-80) were used to produce the stable NEs with Massoia lactone. The results in Table S1 showed that the combination (polyoxyethylene castor + Span-80) could not make the MEs and the components could be easily separated. The combination (Tween-20 + Span-80) could yield the semi-transparent and light blue opalescent NEs with the Tyndall phenomenon and with a particle size of $98 \pm 1.5 \mathrm{~nm}$, PDI value of $0.32 \pm 0.12$ and no phase separation within one week (Table S1). However, the combination (Tween-80 + Span-80) could form stable transparent and light blue opalescent NEs with the Tyndall phenomenon, a smaller particle size of $66 \pm 0.23 \mathrm{~nm}$, PDI value of $0.3 \pm 0.1$ and no phase separation within one week (Table S1). Therefore, finally, in this study, the combination (Tween-80 + Span-80) was used to make the stable NEs with Massoia lactone because the particle size and PDI value were smaller than those of the NEs containing the mixture (Tween-20 + Span-80) (Table S1). Indeed, it has been reported that the sole use of single-chain emulsifiers was less likely to reduce the $\mathrm{o} / \mathrm{w}$ interfacial tension to an appreciable extent. In this case, a co-emulsifier with an amphiphilic nature was used with the emulsifier system [17]. Furthermore, it has been confirmed that the penetration of the co-emulsifiers into the emulsifier interfacial film at the $\mathrm{o} / \mathrm{w}$ interface could further reduce the fluidity of the interface and promote the entropy of the entire colloidal system [17]. This might also happen in the combination (Tween-80 + Span-80) used in this study. In addition, the high molecular weight emulsifier such as Tween- 80 used in this study might make the formed droplets apart by means of steric hindrance because it has been reported that the space necessary for the movement of the polymer chains became restricted and had steric stability in this case [18-20].

In general, NEs are optimized on the basis of particle/globule size, zeta potential (an electrostatic potential that exists very near the surface of particles suspended in liquids) and PDI (polydispersity index, also the stability index in the NEs) [21]. Therefore, the effects of change in ratio of the volume $(\mu \mathrm{L})$ of Tween- 80 to that $(\mu \mathrm{L})$ of Span- 80 on the variation of the droplet size and PDI were examined. The results showed that when the ratios of the volume $(\mu \mathrm{L})$ of Tween-80 to that $(\mu \mathrm{L})$ of Span-80 were 1:9, 2:8 and 3:7, no stable NEs could be formed and the phase separation of the emulsion easily occurred (data not shown). The results in 
Figure 1 A showed that when the ratios of the volume $(\mu \mathrm{L})$ of Tween-80 to that $(\mu \mathrm{L})$ of Span80 were 4:6, 5:5, 6:4, 7:3, 8:2 and 9:1, the stable NEs could be formed and the phase separation of the emulsion did not occur. Especially, when the ratio of the volume $(\mu \mathrm{L})$ of Tween-80 to that $(\mu \mathrm{L})$ of Span-80 was 7:3, the particle size of the formed NEs was $55.0 \pm 0.2 \mathrm{~nm}$ and PDI value was $0.21 \pm 0.02$ (Figure 1 ). So, the ratio (7:3) of the volume $(\mu \mathrm{L})$ of Tween-80 to that $(\mu \mathrm{L})$ of Span-80 was used in the subsequent investigation. It has been reported that a combination of surfactants such as Tween 80/alginate/chitosan [22], lecithin/chitosan [23] sodium dodecyl sulfate (SDS)/chitosan/pectin [24] and $\beta$-lactoglobulin/chitosan/pectin or alginate [25] have been exploited well to develop multi-component biopolymer-based NEs. Therefore, the compositions of the NEs prepared in this study were completely different from those of the NEs prepared by any other researchers.

Then, the ratios of the volume of the mixture (Tween-80: Span-80 =7:3) to that $(\mu \mathrm{L})$ of Massoia lactone were optimized. The results indicated that when the ratios of the volume of the mixture (Tween-80: Span-80 = 7:3) to that $(\mu \mathrm{L})$ of Massoia lactone were 1:9, 2:8, 3:7, $4: 6$ and $5: 5$, no stable NEs could be yielded and the phase separation of the emulsion easily occurred (data not shown). Only the ratios of the volume $(\mu \mathrm{L})$ of the mixture (Tween80: Span-80 = 7:3) to that $(\mu \mathrm{L})$ of Massoia lactone were 6:4, 7:3, 7.5:2.5, 8:2, 8.5:1.5 and $9: 1$, the stable NEs could be obtained and the phase separation of the emulsion did not happen (Figure $1 \mathrm{~B})$. However, when the ratio of the volume $(\mu \mathrm{L})$ of the mixture (Tween-80: Span-80 =7:3) to that $(\mu \mathrm{L})$ of Massoia lactone was 8:2, very stable NEs could be obtained. In this case, the particle size and PDI value of the NEs were $66 \pm 0.23 \mathrm{~nm}$ and $0.3 \pm 0.1$, respectively and the prepared NEs were transparent and light blue opalescent NEs with the Tyndall phenomenon. Furthermore, the further treatment of the system using the ultrasonic power $180 \mathrm{~W}$ and the ultrasonic frequency $22 \mathrm{KHz}$ of the ultrasonic generator were the most suitable for NE formation. Under this condition, when the ultrasonic treatment time was $5 \mathrm{~min}$, the particle size and PDI were $50.1 \mathrm{~nm}$ and 0.19 , respectively (Figure 1C). Based on all the results in Figure 1, the best ingredients for the stable NEs making were $560.0 \mu \mathrm{L}$ of Tween-80, $240.0 \mu \mathrm{L}$ of Span-80 and $200.0 \mu \mathrm{L}$ of Massoia lactone. Then, $9.0 \mathrm{~mL}$ distilled water was titrated into the mixture under continuous magnetic stirring (750 rotations $\mathrm{min}^{-1}$ ) with a speed of about 2 drops of distilled water per second. Finally, the system was treated by ultrasonication using an ultrasonic generator (180 W and $22 \mathrm{KHz}$ ) for $5 \mathrm{~min}$. Indeed, it has been reported that the suitable ultrasonic waves were also used to create the cavitation bubble that collapsed and locally released a substantial amount of energy into the system which could generate the smaller droplets of the internal phase with uniform distribution [25-28].

The stable NEs formed were shown in Figure S1. From the results in Figure S1, it can be clearly observed that the stable NEs were semi-transparent and light blue opalescent (Figure S1A). At the same time, they had the Tyndall phenomenon in the presence of laser light (Figure S1B). Meanwhile, the particle size and morphology of the Massoia lactoneloaded NEs were examined using the Transmission Electron Microscope as described in Materials and methods. It can be clearly seen from Figure 2 that all the particles were spherical, had uniform size distribution and were equally distributed in the NEs. It also can be seen from Figure 2 that the average particle size was $316 \mathrm{~nm}$ which was consistent with that of the particle size shown in Figure 1. It has been reported that the droplet size and droplet distribution were also studied using Dynamic Light Scattering (DLS) and Transmission Electron Microscopy (TEM) [15]. The authors thought that the formed NEs should be a stable system during long-term storage at different temperatures, had extremely small droplet size (20 to $400 \mathrm{~nm}$ ) and had uniform size distribution [15]. The Massoia lactone-loaded NEs prepared in this study indeed could meet such demands (Figures 1 and 2). 


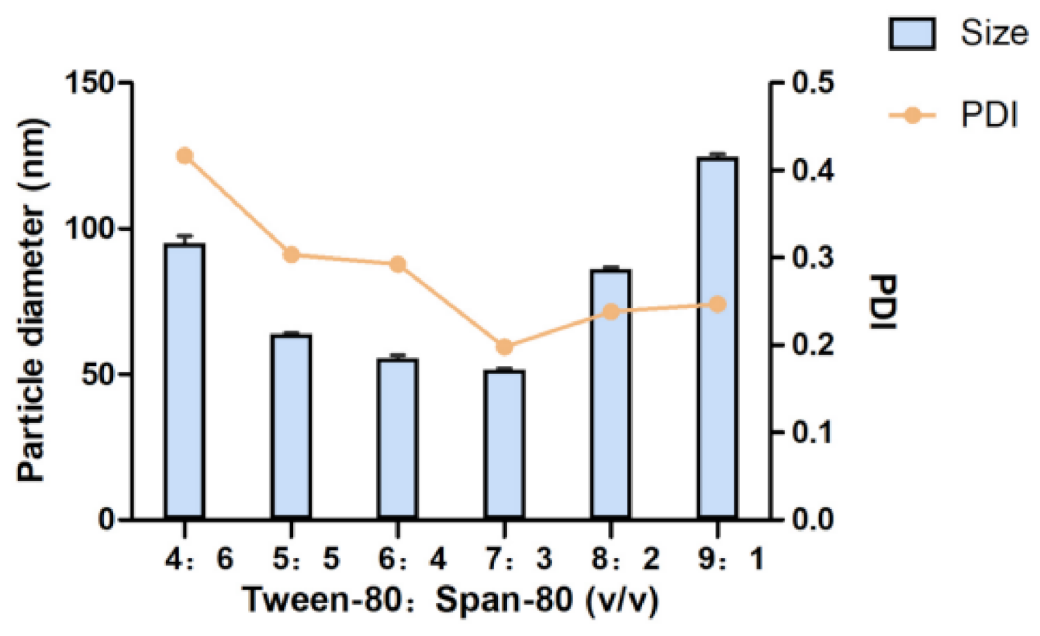

(A)

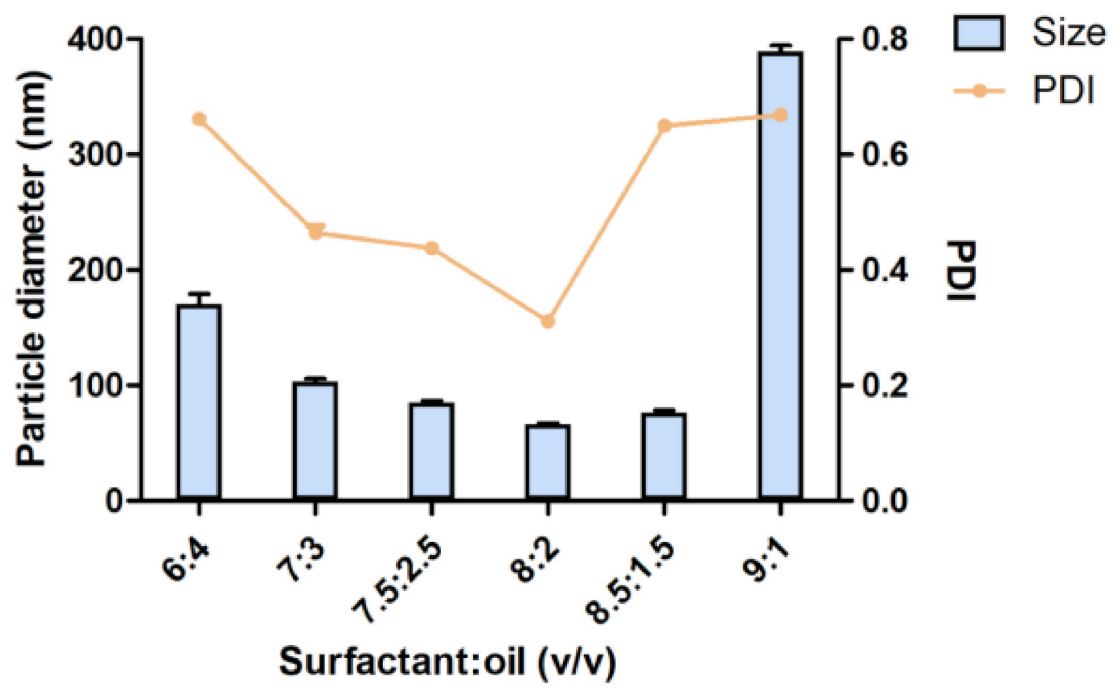

(B)

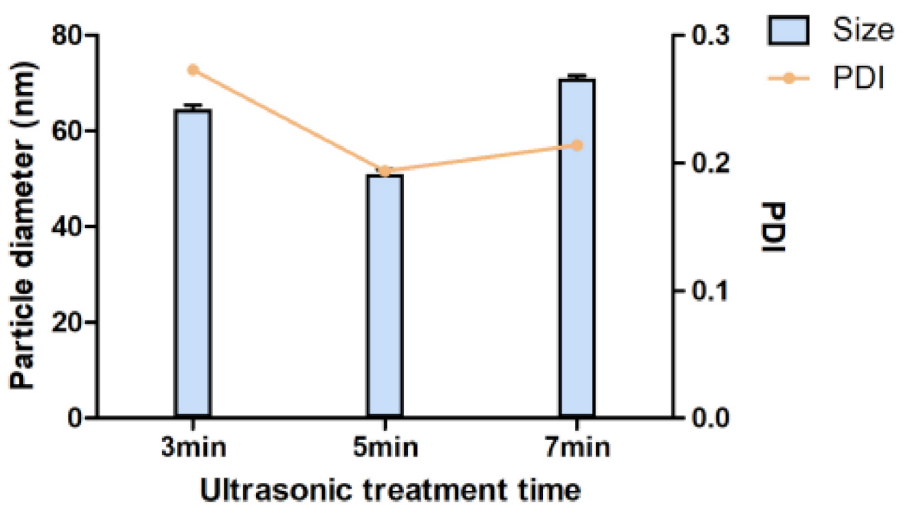

(C)

Figure 1. Influences of ratios of the Tween-80 to Span-80 (A), ratios of surfactants to Massoia lactone (oil) (B) and ultrasonic treatment time (C) on the particle size and PDI of the formed NEs. Data are given as mean $\pm \mathrm{SD}, n=3$. 


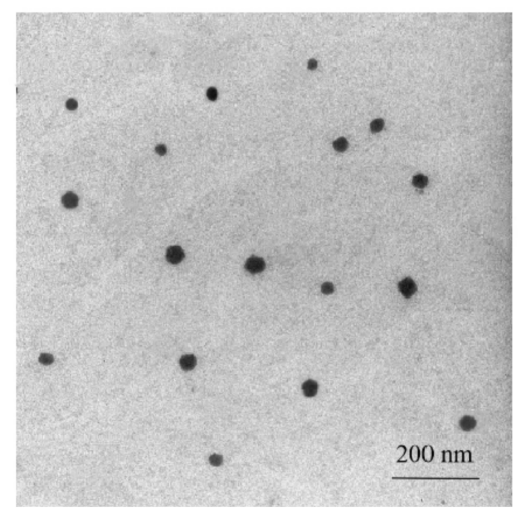

Figure 2. The particles of the Massoia lactone-loaded NEs observed under transmission electron microscope.

\subsection{Stability of the Prepared Massoia Lactone-Loaded NEs}

3.2.1. Stability of the Prepared Massoia Lactone-Loaded NEs during the Storage at Different Temperatures

It was found that after the prepared Massoia lactone-loaded NEs were exposed to $4{ }^{\circ} \mathrm{C}, 25^{\circ} \mathrm{C}, 30^{\circ} \mathrm{C}, 37^{\circ} \mathrm{C}$ and $50^{\circ} \mathrm{C}$ for one week, although they were stable and no phase separation occurred, the colors of them stored at $37^{\circ} \mathrm{C}$ and $50^{\circ} \mathrm{C}$ were changed (data not shown). Therefore, the Massoia lactone-loaded NEs were stored at $4{ }^{\circ} \mathrm{C}, 25^{\circ} \mathrm{C}$ and $30^{\circ} \mathrm{C}$ for 15 and 30 days, respectively and the changes in the particle size and PDI value (0.21) were monitored. The results in Figure 3 indicated that the particle size (diameter: $55 \mathrm{~nm}$ ) and PDI value (0.21) of the Massoia lactone-loaded NEs stored at $4{ }^{\circ} \mathrm{C}$ were not changed. However, as the temperature was increased from $4{ }^{\circ} \mathrm{C}$ to $30^{\circ} \mathrm{C}$, the particle size and PDI value of the Massoia lactone-loaded NEs were continuously increased (Figure 3). Albeit these happened, the droplet size was still kept small (less than $82 \mathrm{~nm}$ ) and the Massoia lactone-loaded NEs were still kept stable. It also has been observed that the key stability issues viz. flocculation, coalescence, creaming, sedimentation and Ostwald ripening of the NEs could be seen in some cases during making and storage of the NEs [15]. However, all the key stability issues did not occur during the storage of the prepared Massoia lactone NEs in this study for 30 days at various temperatures. This meant that the prepared Massoia lactone-loaded NEs indeed were stable during the storage at different temperatures.

\subsubsection{Stability of the Prepared Massoia Lactone-Loaded NEs during the Centrifugation Process}

First, the prepared Massoia lactone NEs were stored at $4{ }^{\circ} \mathrm{C}$ for $7,15,30$ and 45 days. Then, they were centrifuged at $3000 \times g$ and $4000 \times g$ for $15 \mathrm{~min}$. It was found that after the centrifugations, there were no flocculation, coalescence, creaming, sedimentation, Ostwald ripening and phase separation in the prepared Massoia lactone NEs (data not shown). This indicated that the centrifugal stability of the NEs was satisfactory. It has been reported centrifugation of the NE (1000-7000 rpm) could ensure kinetic stability when the NE was studied in terms of creaming, sedimentation, coalescence and phase separation [29]. The cinnamaldehyde, eugenol and carvacrol nanoemulsion which have antifungal activity against Penicillium digitatum were also incorporated into the oil in water NEs and the prepared NEs also had uniform particle size along with good centrifugal stability [16].

\subsubsection{Stability of the Prepared Massoia Lactone-Loaded NEs during the Dilution Process}

First, after the prepared Massoia lactone-loaded NEs were diluted for 2, 5, 8, 10 and 12 folds, the dilutes were stored for one week. Then, the particle sizes were examined. The results in Figure S2 showed that as the dilution folds were increased from 2 to 8, the particle size was continuously decreased. However, as the dilution folds were increased from 5 to 12, the particle size was increased again (Figure S2). After storage for one week, it was found that the NEs were still semi-transparent, light blue opalescent, had the Tyndall 
phenomenon in the presence of laser light and there was no change in the particle size (data not shown). This revealed again that the prepared Massoia lactone loaded NEs were stable during the dilution process.

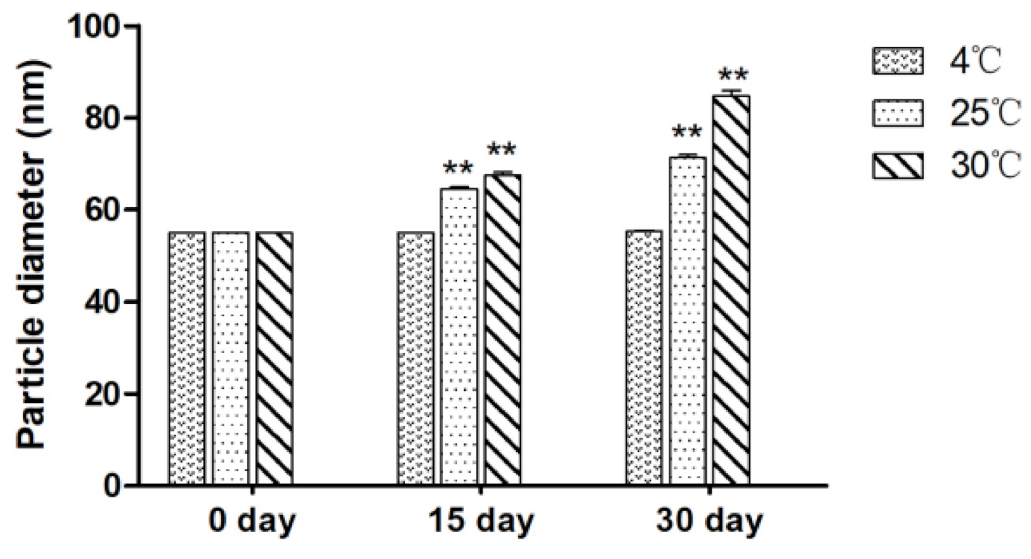

(A)

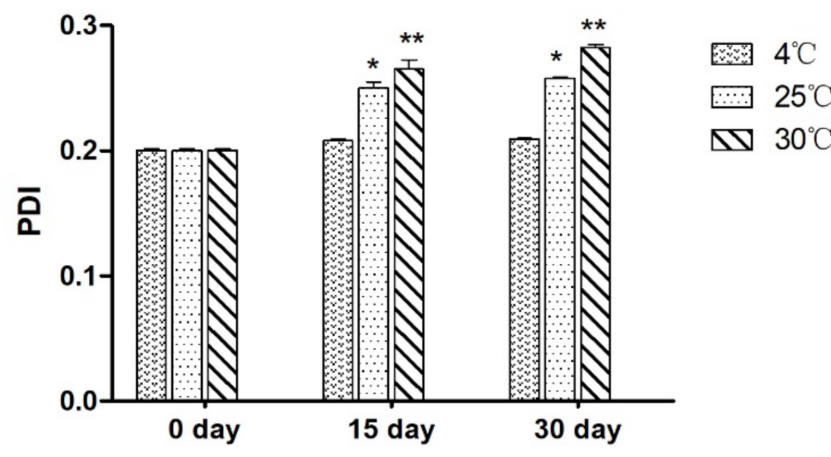

(B)

Figure 3. Effect of different temperatures on the particle size (A) and PDI (B) during storage for 15 and 30 days at temperatures 4,25 and $30^{\circ} \mathrm{C}$. Data are given as mean $\pm \mathrm{SD}, n=3$. Compared with the size and PDI at the beginning, ${ }^{*}(p<0.05)$ means the difference. ${ }^{* *}(p<0.01)$ means the significant difference.

\subsection{Anti-Fungal Activity of the Massoia Lactone-Loaded NEs}

It has been well confirmed that Massoia lactone has high anti-fungal activity against a broad range of yeasts and filamentous fungi [1,4]. So, it is very important to know if Massoia lactone-loaded in the NEs still has such bioactivity. The results in Figure 4A showed that Massoia lactone $\left(20 \mathrm{mg} \mathrm{mL}^{-1}\right)$ in the NEs had the same anti-fungal activity (the diameters of the clear zones were $1.3 \pm 0.1 \mathrm{~cm}$ and $1.3 \pm 0.0 \mathrm{~cm}$, respectively) against the pathogenic yeast M. bicuspidate LIAO in Eriocheir sinensis as free Massoia lactone $(20.0 \mathrm{mg} / \mathrm{mL})$, while, as expected, Tween-80 and Span- 80 had no antifungal activity against the pathogenic yeast $M$. bicuspidate LIAO. However, because Massoia lactone $\left(20.0 \mathrm{mg} \mathrm{mL}^{-1}\right)$ in the NEs could be easily suspended in the liquid and dispersed in any other media, the Massoia lactone-loaded NEs had more potential applications than the free and hydrophobic Massoia lactone. This meant that the Massoia lactone contained in the NEs still kept high antifungal activity. In contrast, the activity of spearmint oil that inhibits oral cancer cells can be increased when it is incorporated into the NEs [30]. Indeed, the oil in water NEs containing cinnamaldehyde, eugenol and carvacrol have significant antifungal activity against $P$. digitatum spore germination, mycelial morphology and membrane permeability and can be used for prolonging the storage period of citrus fruits [16]. 


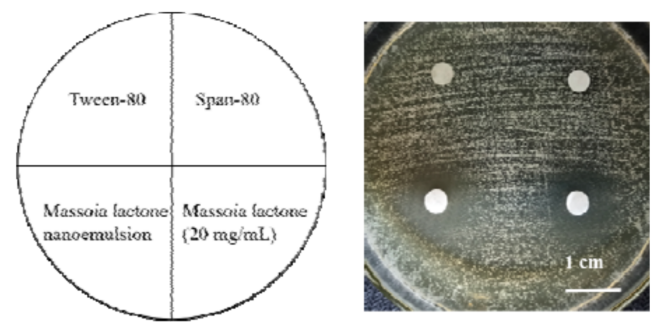

(A)
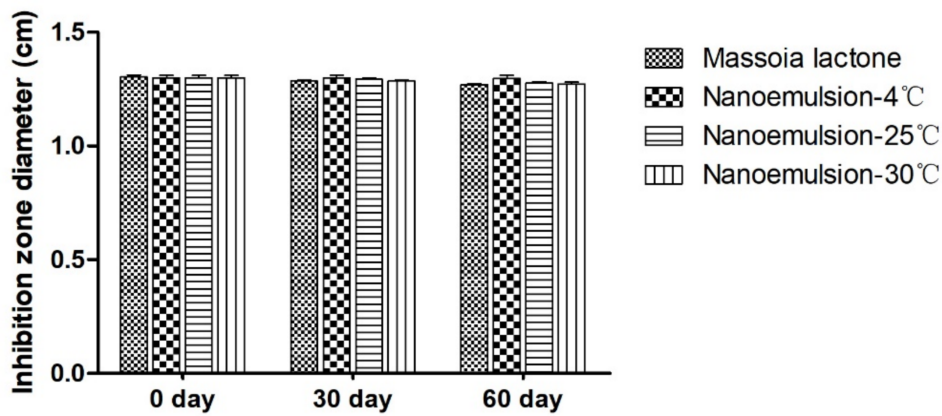

(B)

Figure 4. Antifungal activity (A) and stability of anti-fungal activity (B) of the Massoia lactone loaded NEs and free Massoia lactone. Data are given as mean $\pm \mathrm{SD}, n=3$.

After the Massoia lactone-loaded NEs were stored at $4{ }^{\circ} \mathrm{C}, 25^{\circ} \mathrm{C}$ and $30^{\circ} \mathrm{C}$ for 30 and 60 days, the anti-fungal activity of Massoia lactone loaded in the NEs and free Massoia lactone was measured. It could be observed from the results in Figure $4 \mathrm{~B}$ that there were no changes in the anti-fungal activity (the diameters of the clear zones were kept almost the same) of Massoia lactone loaded in the NEs and free Massoia lactone during the storage at $4{ }^{\circ} \mathrm{C}, 25^{\circ} \mathrm{C}$ and $30^{\circ} \mathrm{C}$ for 30 and 60 days. This demonstrated that the used Massoia lactone was very stable under different conditions and had highly potential applications in the food industry. It has been reported that the prepared NEs can be used to deliver and disperse flavors, fragrances, natural and synthetic drugs [15]. Therefore, the Massoia lactone loaded NEs prepared in this study will also be used to deliver and disperse Massoia lactone for killing the pathogenic fungi in the future.

\subsection{Anti-Oxidant Activity of the Massoia Lactone Loaded NEs}

The results in Figure S3 showed that both free Massoia lactone and the Massoia lactone loaded in the NEs had almost the same DPPH radical scavenging activity and the hydroxyl radical scavenging activity. For example, free Massoia lactone and the Massoia lactone loaded in the NEs had the DPPH radical scavenging rates of $36.1 \%$ and $28.9 \%$, respectively (Figure S3) while free Massoia lactone and the Massoia lactone loaded in the NEs had the hydroxyl radical scavenging rates of $35.3 \%$ and $28.1 \%$, respectively (Figure S3). This meant that in addition to its high anti-fungal activity (Figure 4), Massoia lactone also had antioxidant activity and indeed could have pharmacological effects. However, the mechanisms of the antioxidant activity of Massoia lactone are still completely unknown. It has been well documented that reactive oxygen species (ROS), including superoxide $\left(\mathrm{O}^{2-}\right)$, hydroxyl (HO), peroxyl (ROO), alkoxyl (RO) and nitricoxide (NO) are closely related to many diseases in animals and human. This meant that Massoia lactone with various bioactivities also had high potential applications in the medical industry. However, many natural products such as vitamin C (Figure S3), vitamin E, carotenoids, red ginseng and flavonoids have much higher DPPH radical scavenging activity and the hydroxyl radical scavenging activity than Massoia lactone used in this study [14,31-33]. Therefore, it is important how to further improve the DPPH radical scavenging activity and the hydroxyl radical scavenging activity of Massoia lactone. 


\subsection{Massoia Lactone Loaded in NEs Could Damage the Cell Membrane and Cause Cellular Necrosis of the Pathogenic Yeast}

It has been confirmed that Massoia lactone as a bio-surfactant could damage the cell membrane, even break the whole cells of the pathogenic yeast strain LIAO and cause cellular necrosis of the pathogenic yeast LIAO [4]. Therefore, the cells of the pathogenic yeast LIAO treated using Massoia lactone loaded in the NEs and stained using PI were observed under the Olympus U-LH100HG fluorescent microscope. The results in Figure 5D indicated that the cells of the pathogenic yeast LIAO treated using Massoia lactone loaded in the NEs were stained to be red by PI while the cells of the pathogenic yeast LIAO untreated using Massoia lactone loaded in the NEs could not be stained by PI (Figure 5B). This meant that the cell membrane integrity of the cells of the pathogenic yeast LIAO treated using Massoia lactone loaded in the NEs was damaged. In our previous study [4], the cell membrane integrity of the cells of the pathogenic yeast LIAO treated using free Massoia lactone was also damaged, suggesting that bioactivity of Massoia lactone loaded in the NEs was not changed.

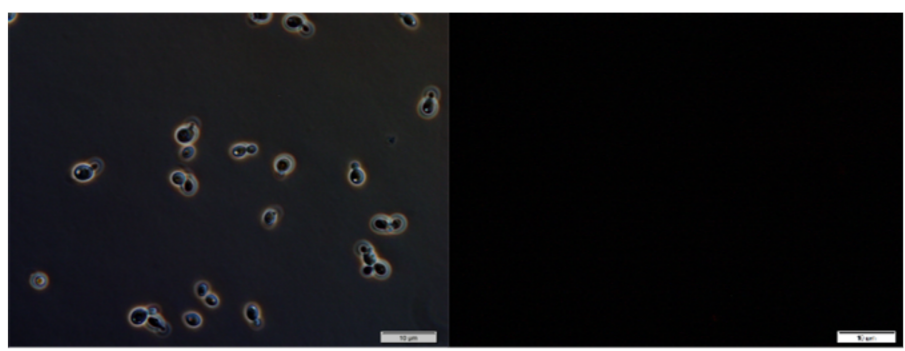

A

B

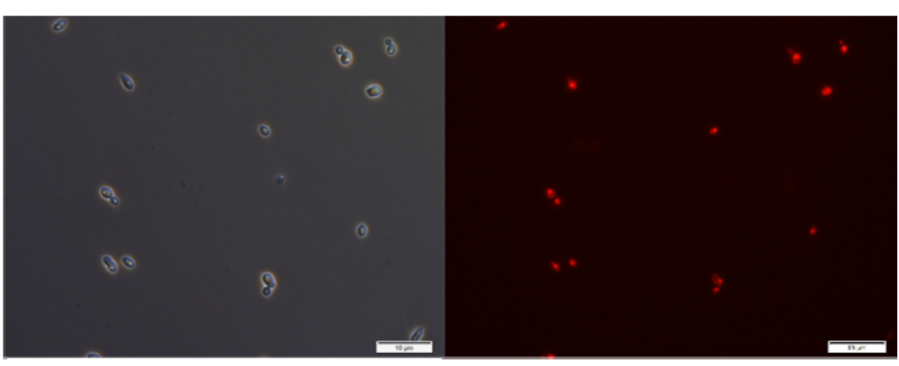

C

D

Figure 5. PI staining of the Massoia lactone NEs untreated pathogenic yeast cells $(\mathbf{A}, \mathbf{B})$ and PI staining of the Massoia lactone NEs treated pathogenic yeast cells (C,D). Photos A and C were taken under the normal light and Photo B was taken under UV light. The Bar $=10 \mu \mathrm{m}$.

At the same time, the cells of the pathogenic yeast LIAO treated using Massoia lactone loaded in the NEs and those of the pathogenic yeast LIAO untreated using Massoia lactone loaded in the NEs were also stained using the Annexin V-FITC apoptosis detection kit as described in Materials and methods. The treated cells and untreated cells were observed and photographed under the Olympus U-LH100HG fluorescent microscope. It can be clearly seen from the results in Figure 6 that the treated cells were stained to be both blue and red (Figure 6E,F) while the untreated cells could not be stained (Figure $6 \mathrm{~B}, \mathrm{C}$ ). It has been well confirmed if the treated pathogenic yeast cells by Massoia lactone were only stained to be blue, not red by the kit used in this study, this meant that the treatment caused apoptosis in the yeast cells. In contrast, if the treated pathogenic yeast cells by Massoia lactone were stained to be both blue and red by the kit used in this study, this meant that the treatment caused necrosis of the pathogenic yeast cells [13]. Therefore, as with free Massoia lactone, the yeast cells treated with the Massoia lactone loaded in the NEs also caused cellular necrosis of the pathogenic yeast LIAO. 


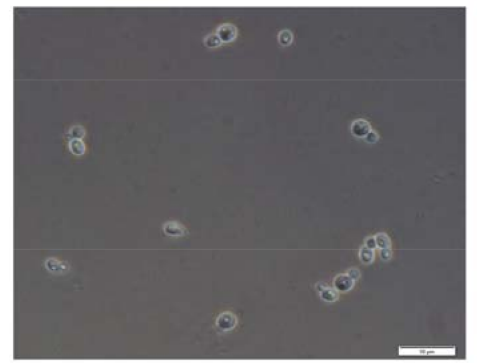

A

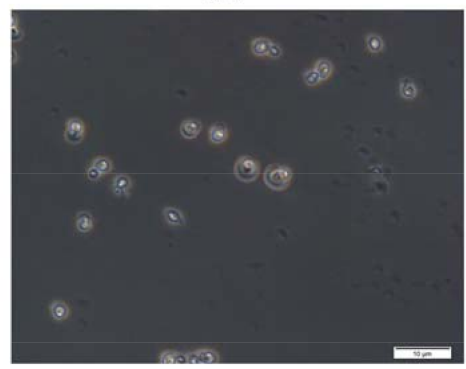

D

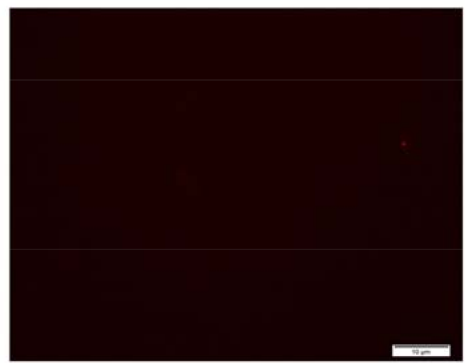

B

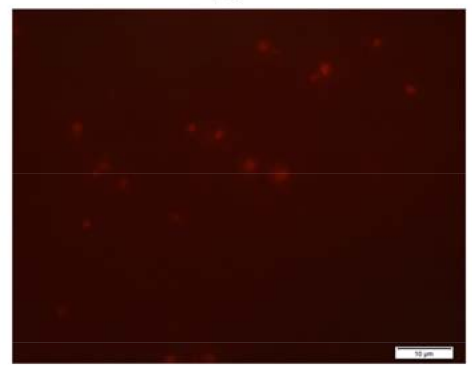

E

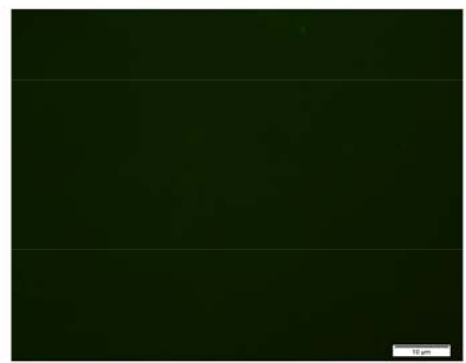

C

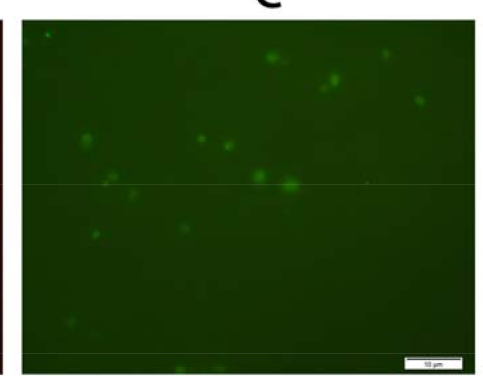

F

Figure 6. PI and Annexin V-FITC staining of the Massoia lactone NEs untreated pathogenic yeast cells $(\mathbf{A}-\mathbf{C})$ and PI staining of the Massoia lactone NEs treated pathogenic yeast cells (D-F). The photos $(\mathbf{A}, \mathbf{D})$ were taken under the normal light and the photos $(\mathbf{B}, \mathbf{C}, \mathbf{E}, \mathbf{F})$ were taken under UV light. The Bar $=10 \mu \mathrm{m}$.

\section{Conclusions}

In summary, Massoia lactone has high potential antifungal activities. Because of the insolubility and weak volatility of Massoia lactone, it should be loaded in NEs. In this study, it was found that the prepared Massoia lactone loaded NEs and their anti-fungal and antioxidant activity were very stable during storage, centrifugation and dilution under different conditions. Massoia lactone loaded in the NEs was easily dispersed in the liquid compared with the free Massoia lactone and could kill the yeast cells by damaging their cell membrane and causing cellular necrosis. Therefore, Massoia lactone loaded NEs could be easily used for effective treatment of pathogenic fungi and yeasts in the food in the future.

Supplementary Materials: The following supporting information can be downloaded at: https:// www.mdpi.com/article/10.3390/jmse10030339/s1, Table S1. Various combinations for making the Massoia lactone loaded NEs. Figure S1. The appearance of the formed Massoia lactone loaded NEs (A) and the observed Tyndall phenomenon of them (B). Figure S2. Stability of the Massoia lactone NEs during dilution. Data are given as mean $\pm \mathrm{SD}, n=3$. Figure S3. The DPPH radical scavenging activity and the hydroxyl radical scavenging activity of free Massoia lactone and Massoia lactone loaded NEs. Data are given as mean $\pm \mathrm{SD}, n=3$. Com-pared with scavenging rate of vitamin $\mathrm{C}$, ** $(p<0.01)$ means the significant difference.

Author Contributions: L.Y. and H.-Q.Z.: Data curation and doing all the experiments, Z.C.: Writing original draft. G.-L.L.: Data curation, Writing—original draft. Z.-M.C.: Supervision. All authors have read and agreed to the published version of the manuscript.

Funding: The research work was supported by the research grants from both National Natural Science Foundation of China and the Fundamental Research Funds for the Central Universities (Grant Nos. 31500029 and 31970058).

Informed Consent Statement: Not applicable.

Data Availability Statement: Not applicable. 
Acknowledgments: Thanks for the financial support by the research grants from both National Natural Science Foundation of China and the Fundamental Research Funds for the Central Universities (Grant Nos. 31500029 and 31970058).

Conflicts of Interest: The authors declared that there is no conflict of interest.

\section{References}

1. Kang, X.X.; Jia, S.L.; Wei, X.; Zhang, M.; Liu, G.L.; Hu, Z.; Chi, Z.; Chi, Z.M. Liamocins biosynthesis, its regulation in Aureobasidium spp., and their Bioactivities. Crit. Rev. Biotechnol. 2022, 42, 93-105. [CrossRef] [PubMed]

2. Xue, S.L.; Liu, G.L.; Chi, Z.; Jiang, H.; Hu, Z.; Chi, Z.M. Genetic evidences for the core489 biosynthesis pathway, regulation, transport and secretion of liamocins in yeast-like fungal cells. Biochem. J. 2020, 477, 887-903. [CrossRef] [PubMed]

3. Saika, A.; Fukuoka, T.; Mikome, S.; Kondo, Y.; Habe, H.; Morita, T. Screening and isolation of the liamocin-producing yeast Aureobasidium melanogenum using xylose as the sole carbon source. J. Biosci. Bioeng. 2020, 129, 428-434. [CrossRef] [PubMed]

4. Zhang, H.Q.; Chi, Z.; Liu, G.L.; Zhang, M.; Hu, Z.; Chi, Z.M. Metschnikowia bicuspidate associated with a milky disease in Eriocheir sinensis and its effectitve treatment by Massoia lactone. Microbiol. Res. 2021, 242, 126641. [CrossRef]

5. $\quad$ Tang, R.R.; Chi, Z.; Jiang, H.; Liu, G.L.; Chi, Z.M. Overexpression of a pyruvate carboxylase gene enhances extracellular liamocin and intracellular lipid biosynthesis by Aureobasidium melanogenum M39. Proc. Biochem. 2018, 69, 64-74. [CrossRef]

6. Kishimoto, N.; Sugihara, S.; Mochida, K. In vitro antifungal and antiviral activities of $\gamma$ - and $\delta$-lactone analogs utilized as food flavoring. Biocontr. Sci. 2005, 10,31-36. [CrossRef]

7. Kurosawa, T.; Sakai, K.; Nakahara, T. Extracellular accumulation of the polyol lipids 3,5-dihydroxydecanoyl and 5-hydroxy-2decenoyl esters of arabitol and mannitol by Aureobasidium sp. Biosci. Biotechnol. Biochem. 1994, 58, 2057-2060. [CrossRef]

8. Barros, M.E.S.B.; Freitas, J.C.R.; Oliveira, J.M. Synthesis and evaluation of (-)-Massoia lactone and analogues as potential anticancer and anti-inflammatory agents. Eur. J. Med. Chem. 2014, 76, 291-300. [CrossRef]

9. Sang, Q.; Pan, Y.; Jiang, Z. HPLC determination of massoia lactone in fermented Cordyceps sinensis mycelium Cs-4 and its anticancer activity in vitro. J. Food Biochem. 2020, 44, e13336. [CrossRef]

10. Soundararajan, V.; Kandasamy, V.; Subramani, P. Antibiofilm, antioxidant and larvicidal activity of formulated nanoemulsion from Ocimum tenuiflorum. Mat. Today Proceed. 2021, 45, 3438-3443. [CrossRef]

11. Rao, J.; McClements, D.J. Food-grade microemulsions and nanoemulsions: Role of oil phase composition on formation and stability. Food Hydrocoll. 2012, 29, 326-334. [CrossRef]

12. Hong, L.; Zhou, C.L.; Chen, F.P.; Han, D.; Wang, C.Y.; Li, J.X. Development of a carboxymethyl chitosan functionalized nanoemulsion formulation for increasing aqueous solubility, stability and skin permeability of astaxanthin using low-energy method. J. Microencapsulat. 2017, 34, 707-721. [CrossRef]

13. Jiang, L.; Zhang, M.; Meng, Z.; Xie, M. Honokiol kills Candida albicans through ROS accumulation and cell membrane destruction. Acta Microbiol. Sin. 2018, 58, 511-519.

14. Shao, L.L.; Xu, J.; Shi, M.J.; Wang, X.L.; Li, Y.T.; Kong, L.M.; Hider, R.C.; Zhou, T. Preparation, antioxidant antimicrobial evaluation of hydroxamated degraded polysaccharides from Enteromorpha prolifera. Food Chem. 2017, 237, 481-487. [CrossRef]

15. Rai, V.K.; Mishra, N.; Yadav, K.S.; Yadav, N.P. Nanoemulsion as pharmaceutical carrier for dermal and transdermal drug delivery: Formulation development, stability issues, basic considerations and applications. J. Contr. Rel. 2018, 270, 203-225. [CrossRef] [PubMed]

16. Yang, R.; Miao, J.; Shen, Y.; Cai, N.; Wan, C.; Zou, L. Antifungal effect of cinnamaldehyde, eugenol and carvacrol nanoemulsion against Penicillium digitatum and application in postharvest preservation of citrus fruit. LWT Food Sci. Technol. 2021, 141, 110924 [CrossRef]

17. Figen, T.; Seyda, A.; Nevin, C. Nanoemulsions as Drug Delivery Systems. In Colloids in Drug Delivery; Fanun, M., Ed.; CRC Press: Boca Raton, FL, USA, 2010; pp. 221-244.

18. Koroleva, M.Y.; Evgenii, V.Y. Nanoemulsions: The properties, methods of preparation and promising applications. Rus. Chem. Rev. 2012, 81, 21. [CrossRef]

19. Liu, W.; Sun, D.; Li, C.; Liu, Q.; Xu, J. Formation and stability of paraffin oil-in-water nano-emulsions prepared by the emulsion inversion point method. J. Colloid Interf. Sci. 2006, 303, 557-563. [CrossRef]

20. Hoeller, S.; Sperger, A.; Valenta, C. Lecithin based nanoemulsions: A comparative study of the influence of non-ionic surfactants and the cationic phytosphingosine on physicochemical behaviour and skin permeation. Internt. J. Pharm. 2009, 370, 181-186. [CrossRef]

21. Pund, S.; Shete, Y.; Jagadale, S. Multivariate analysis of physicochemical characteristics of lipid based nanoemulsifying cilostazolquality by design. Colloids Surf. B Biointerf. 2014, 115, 29-36. [CrossRef]

22. Mishra, N.; Yadav, K.S.; Rai, V.K.; Yadav, N.P. Polysaccharide encrusted multilayered nano-colloidal system of andrographolide for improved hepatoprotection. AAPS Pharm. Scient. Technol. 2017, 18, 381-392. [CrossRef]

23. Klinkesorn, U.; McClements, D.J. Influence of chitosan on stability and lipase digestibility of lecithin-stabilized tuna oil-in-water emulsions. Food Chem. 2009, 114, 1308-1315. [CrossRef] 
24. Aoki, T.; Decker, E.A.; McClements, D.J. Influence of environmental stresses on stability of O/W emulsions containing droplets stabilized by multilayered membranes reduced by a layer-by-layer electrostatic deposition technique. Food Hydrocol. 2005, 19, 209-220. [CrossRef]

25. Li, Y.; Hu, M.; Xiao, H.; Du, Y.; Decker, E.A.; McClements, D.J. Controlling the functional performance of emulsion-based delivery systems using multi-component biopolymer coatings. Eur. J. Pharm. Biopharma. 2010, 76, 38-47. [CrossRef]

26. Sutradhar Kumar, B.; Amin, L. Nanoemulsions: Increasing possibilities in drug delivery. Eur. J. Nanomed. 2013, 97, 237-245. [CrossRef]

27. Sugumar, S.; Ghosh, V.; Nirmala, M.L.; Mukherjee, A.; Chandrasekaran, N. Ultrasonic emulsification of eucalyptus oil nanoemulsion: Antibacterial activity against Staphylococcus aureus and wound healing activity in Wistar rats. Ultrason. Sonochem. 2014, 21, 1044-1049. [CrossRef] [PubMed]

28. Laxmi, M.; Bhardwaj, A.; Mehta, S.; Mehta, A. Development and characterization of nanoemulsion as carrier for the enhancement of bioavailability of artemether. Artif. Cell. Nanomed. Biotechnol. 2014, 43, 334-344. [CrossRef]

29. Singh, Y.; Meher, J.G.; Raval, K.; Khan, F.A.; Chaurasia, M.; Jain, N.K. Nanoemulsion: Concepts, development and applications in drug delivery. J. Cont. Relea. 2017, 252, 28-49. [CrossRef]

30. Tubtimsri, S.; Limmatvapirat, C.; Limsirichaikul, S.; Akkaramongkolporn, P.; Piriyaprasarth, S.; Patomchaiviwat, V. Incorporation of fixed oils into spearmint oil-loaded nanoemulsions and their influence on characteristic and cytotoxic properties against human oral cancer cells. J. Drug Deliv. Sci. Technol. 2021, 63, 102443. [CrossRef]

31. Gulcin, I. Antioxidant activity of food constituents: An overview. Arch. Toxicol. 2012, 86, 345-391. [CrossRef]

32. Schlesier, K.; Harwat, M.; Bohm, V. Assessment of antioxidant activity by using different in vitro methods. Free Rad. Res. 2002, 36, 237-242. [CrossRef] [PubMed]

33. Dawidowicz, A.L.; Olszowy, M.; Magorzata, J.D. Importance of solvent association in the estimation of antioxidant properties of phenolic compounds by DPPH method. J. Food Sci. Technol. 2015, 52, 4523-4529. [CrossRef] [PubMed] 